\title{
FACTORES ASOCIADOS A MORTALIDAD INTRAHOSPITALARIA DE UNA POBLACIÓN EN HEMODIÁLISIS EN EL PERÚ
}

\author{
Percy Herrera-Añazco ${ }^{1,2, a}$, Vicente A. Benítes-Zapata ${ }^{3,4, b}$, Adrián V. Hernandez ${ }^{5, c}$
}

\begin{abstract}
RESUMEN
Objetivos. Determinar los factores asociados a la mortalidad durante la primera hospitalización de una población incidente en hemodiálisis. Materiales y métodos. Estudio observacional y retrospectivo de pacientes que ingresaron al Hospital Nacional Dos de Mayo entre enero de 2012 y diciembre de 2013. Para el análisis de la supervivencia utilizamos el método de Kaplan-Meier. Se realizó un análisis de regresión logístico multivariado para evaluar los factores asociados a mortalidad intrahospitalaria. Resultados. Se estudiaron 216 pacientes con edad promedio de 56,9 $\pm 15,5$ años. El $24 \%$ de los paciente $(n=51)$ fallecieron durante la estancia hospitalaria. La tasa de mortalidad fue de 9,3 muertes/100 personas-semanas (IC 95\%: 7,0 a 12,3). Se evidenció una tendencia a menor riesgo de fallecer en pacientes que tenían entre uno y seis meses con diagnóstico de enfermedad renal crónica (OR 0,84; IC 95\%: 0,32 a 2,26), y en aquellos con más de seis meses comparado con aquellos que lo tenían hace menos de un mes (OR 0,55; IC 95\%: 0,19 a 1,57). La atención previa por un nefrólogo no estuvo asociada a diferencias en la mortalidad menor (OR 1,14; IC 95\%: 0,39 a $3,31)$. Conclusiones. Existe una deficiente atención previa entre los pacientes en hemodiálisis que forman parte de una inadecuada estructura de atención de salud y que está asociado a una alta mortalidad intrahospitalaria.
\end{abstract}

Palabras clave: Mortalidad; Hemodiálisis; Sistemas de salud (fuente: DECS/Bireme)

\section{FACTORS ASSOCIATED WITH IN HOSPITAL DEATHS IN A HEMODIALYSIS POPULATION IN PERU}

\begin{abstract}
Objectives. To determine the factors associated with mortality during the first hospitalization of patients admitted to a hemodialysis unit. Materials and methods. Observational and retrospective study of patients admitted to "Dos de Mayo" National Hospital between January 2012 and December 2013. For the survival analysis we used the Kaplan-Meier method. A multivariate logistic regression was performed to evaluate the factors associated with hospital mortality. Results. 216 patients with a mean age of $56.9 \pm 15.5$ years were studied. $24 \%$ of patients $(n=51)$ died during their hospital stay. The mortality rate was 9.3 deaths $/ 100$ person-weeks $(95 \% \mathrm{Cl}: 7.0$ to 12.3$)$. We found a tendency of less risk of death in patients with between 1 and 6 months from chronic kidney disease diagnosis (OR 0.84, 95\% Cl: 0.32 to 2.26) and in those with more than six months from chronic kidney disease diagnosis compared with those who had less than a month from chronic kidney disease diagnosis (OR $0.55,95 \% \mathrm{Cl}: 0.19$ to 1.57 ). Previous care by a nephrologist was not associated with differences in lower mortality (OR $1.14,95 \% \mathrm{Cl}: 0.39$ to 3.31 ). Conclusions. There is poor prior care among hemodialysis patients that form part of an inadequate health care structure and this is associated with high inhospital mortality.
\end{abstract}

Key words: Mortality; Hemodialysis; Health systems (source: MeSH/NLM)

\section{INTRODUCCIÓN}

La mortalidad anual de los pacientes en hemodiálisis (HD) es alta ${ }^{(1,2)}$, siendo mayor en los primeros tres meses (3). Sin embargo, se ha descrito que la mortalidad en los primeros días del inicio de la terapia está subregistrada, lo que en Estados Unidos (EE. UU.) se ha atribuido a una falta de estímulo para la misma en términos de reembolso económico ${ }^{(4)}$.

Los factores asociados a la mortalidad varían dependiendo del momento en que esta se registra. Se

\footnotetext{
Hospital Nacional Dos de Mayo. Lima, Perú.

Facultad de Medicina Humana, Universidad Nacional de Piura. Piura, Perú.

Unidad de Análisis y Generación de Evidencias en Salud Pública, Instituto Nacional de Salud. Lima, Perú.

Facultad de Medicina Humana. Universidad de San Martín de Porres. Lima, Perú

Escuela de Medicina, Universidad Peruana de Ciencias Aplicadas. Lima, Perú

Medico nefrólogo, magíster en docencia en Educación Superior; ${ }^{\mathrm{b}}$ médico auditor; ${ }^{\mathrm{c}}$ médico, doctor en Epidemiología Clínica

Recibido: : 20-10-14 Aprobado: 18-03-15
}

Citar como: Herrera-Añazco P, Benítes-Zapata V, Hernandez AV. Factores asociados a mortalidad intrahospitalaria de una población en hemodiálisis en el Perú. Rev Peru Med Exp Salud Publica. 2015;32(3):479-84. 
ha sugerido que los factores asociados a la mortalidad temprana dependerían de las condiciones clínicas en las que el paciente inicia la terapia; esto indirectamente está relacionado con la estructura de la atención de los pacientes con enfermedad renal crónica (ERC) en un sistema de salud ${ }^{(5,6)}$.

El Ministerio de Salud del Perú (MINSA) no cuenta con un programa de atención integral para los pacientes con ERC, y entre los pacientes que requieren algún tipo de terapia de reemplazo renal (TRR) la cobertura es limitada, habiéndose reportado una alta frecuencia de retiro por falta de cupos disponibles ${ }^{(7,8)}$. Si bien en nuestro país se ha descrito una alta mortalidad entre pacientes en HD durante su primera hospitalización ${ }^{(9)}$, no se han estudiado los factores asociados a ella; este análisis podría desnudar falencias en la estructura de atención de los pacientes con ERC en el MINSA y deberían ser remediadas en los planes de reformas de salud en nuestro país.

En este estudio determinamos los factores asociados a la mortalidad durante la primera hospitalización de la población incidente en diálisis en un hospital de referencia nacional del MINSA.

\section{MATERIALES Y MÉTODOS}

Se llevó a cabo un estudio observacional, retrospectivo y analítico en una cohorte de pacientes incidentes con ERC que ingresaron por emergencia del Hospital Nacional Dos de Mayo (HN2M) de Lima, para su primera HD entre el 1 de enero de 2012 al 31 de diciembre de 2013. Se excluyó a pacientes con ERC que ingresaron con HD en forma ambulatoria y programada, y a los pacientes que hubieran iniciado HD en otro hospital.

Las variables demográficas como edad y sexo, y las variables clínicas como tiempo con diagnóstico de ERC, etiología de la ERC y el tipo de acceso vascular utilizado, se recolectaron de los registros de vigilancia epidemiológica del servicio de nefrología. Así mismo, se registraron exámenes de laboratorio como tasa de filtrado glomerular (TFG) estimada mediante la fórmula de Modification Dialysis Renal Disease 4 (MDRD4) ${ }^{(10)}$, hemoglobina, potencial de hidrógeno $(\mathrm{pH})$, bicarbonato $\left(\mathrm{HCO}^{3}\right)$, calcio $(\mathrm{Ca})$, fosforo $(\mathrm{P})$ y potasio $(\mathrm{K})$, los cuales fueron recolectados previos al inicio de la primera HD.

Se definió anemia, a la presencia de hemoglobina (Hb) menor a $13 \mathrm{~g} / \mathrm{dL}$, y anemia severa si la $\mathrm{Hb}$ era menor a $7 \mathrm{~g} / \mathrm{dL}$. Se definió como acidosis a un $\mathrm{HCO}^{3}$ menor de $24 \mathrm{mEq} / \mathrm{L}$; hiperkalemia a un $\mathrm{K}$ mayor de $5,5 \mathrm{mEq} / \mathrm{L}$; hipocalcemia a un $\mathrm{Ca}$ menor a $8,5 \mathrm{mg} /$ $\mathrm{dL}$, e hiperfosfatemia a un $\mathrm{P}$ mayor a $5,5 \mathrm{mg} / \mathrm{dL}$. Los exámenes auxiliares se procesaron en el laboratorio central del HN2M. Para la medición de la creatinina se usó el método de Jaffe; para la medición de $\mathrm{Ca}, \mathrm{P}$ y electrolitos se usó la técnica de química seca; para la hemoglobina se usó el método de sulfato de laurel de sodio de Sysmex (SLS), y para la medición de gases arteriales se usó el método de potenciometría mediante electrodos.

Adicionalmente, se determinó si los pacientes habían sido atendidos previamente en el HN2M y si habían sido evaluados por un nefrólogo. Se consideró paciente del HN2M a aquel que tenía historia clínica previa en el hospital. El resto de pacientes eran considerados "de otros hospitales", aunque podría existir la posibilidad de que no haya tenido ninguna evaluación médica previa ya que ese dato no se especifica en los registros de vigilancia epidemiológica del servicio de nefrología. Se definió como "paciente evaluado por un nefrólogo" a todo paciente que fue atendido, por lo menos, dos veces en el último año en consultorio de nefrología. Los pacientes fueron seguidos desde su ingreso por emergencias hasta la fecha del alta. Así mismo, se determinó en ellos su estado vital al alta hospitalaria.

Las variables continuas son presentadas como promedios con desviación estándar o como mediana con rango intercuartílico (RIC) si tenían distribuciones sesgadas. Las variables categóricas son presentadas como frecuencias y porcentajes. Para comparar variables continuas se usó la prueba de T de Student o la prueba U de Mann-Whitney cuando las variables tenían distribución sesgada. Para comparar variables categóricas se usó la prueba de chi cuadrado. Para el análisis de la función de supervivencia se empleó el método de Kaplan-Meier y la prueba log rank test. Para valorar la asociación de algunas variables con la mortalidad intrahospitalaria, se llevó a cabo un modelo de regresión logística multivariado definido a priori, que incluyó edad, etiología de ERC, tiempo con el diagnóstico de ERC, atención previa por nefrólogo y TFG. En el modelo, la variable TFG fue transformada a logaritmo de TFG por tener distribución sesgada. Se presenta como medida de asociación al Odds Ratio (OR) con su intervalo de confianza (IC) al 95\%. Los análisis fueron realizados con el paquete estadístico STATA (Statacorp, TX, USA) versión 12.1. Los datos de este estudio se obtuvieron de los registros empleados en la vigilancia epidemiológica del servicio de nefrología del HN2M, por lo que no fue necesario someterlo al comité de ética del hospital.

\section{RESULTADOS}

Durante el periodo de estudio se recolectaron 235 pacientes, de los cuales 19 fueron excluidos debido a que ingresaron a diálisis en forma ambulatoria y programada. Los 216 pacientes incluidos tuvieron un 
Tabla 1. Características clínicas-demográficas de los participantes al estudio en general y según estado vital

\begin{tabular}{|c|c|c|c|c|}
\hline Características & $\begin{array}{c}\text { Total = } 216 \\
(\%)\end{array}$ & $\begin{array}{c}\text { Muerto }=51 \\
(\%)\end{array}$ & $\begin{array}{c}\text { Vivo }=165 \\
(\%)\end{array}$ & Valor $p$ \\
\hline$\overline{\text { Edad (años)* }}$ & $56,9 \pm 15,5$ & $58,6 \pm 14,9$ & $56,3 \pm 15,6$ & 0,35 \\
\hline Sexo masculino & $134(62,0)$ & $30(58,8)$ & $104(63,0)$ & 0,59 \\
\hline \multicolumn{5}{|l|}{ Etiología de ERC } \\
\hline Nefropatía diabética & $69(31,9)$ & $21(41,18)$ & $48(29,1)$ & 0,21 \\
\hline Glomerulonefritis crónica & $38(17,6)$ & $10(19,61)$ & $28(16,9)$ & \\
\hline Uropatía obstructiva & $27(12,5)$ & $3(5,9)$ & $24(14,6)$ & \\
\hline Nefropatía hipertensiva & $20(9,3)$ & $3(5,9)$ & $17(10,3)$ & \\
\hline Poliquistosis renal & $9(4,2)$ & $1(1,96)$ & $8(4,9)$ & \\
\hline No reportada & $53(24,5)$ & $13(25,5)$ & $40(24,2)$ & \\
\hline \multicolumn{5}{|l|}{ Tiempo del diagnóstico } \\
\hline$<1$ mes & $98(45,4)$ & $24(47,1)$ & $74(44,9)$ & 0,76 \\
\hline 1 a 6 meses & $50(23,2)$ & $13(25,5)$ & $37(22,4)$ & \\
\hline$>6$ meses & $68(31,5)$ & $14(27,5)$ & $54(32,7)$ & \\
\hline \multicolumn{5}{|l|}{ Tipo de acceso vascular } \\
\hline Fistula arteriovenosa & $6(2,8)$ & $0(0)$ & $6(3,7)$ & 0,17 \\
\hline Catéter no tunelizado & $207(97,2)$ & $50(100)$ & $157(96,3)$ & \\
\hline \multicolumn{5}{|l|}{ Procedencia } \\
\hline HN2M & $73(33,8)$ & $24(47,1)$ & $49(29,7)$ & 0,03 \\
\hline Otros hospitales & $143(66,2)$ & $27(52,9)$ & $116(70,3)$ & \\
\hline \multicolumn{5}{|l|}{ Atención previa por nefrólogo } \\
\hline Sí & $46(21,3)$ & $12(23,5)$ & $34(20,6)$ & 0,66 \\
\hline No & $170(78,7)$ & $39(76,5)$ & $131(79,4)$ & \\
\hline \multicolumn{5}{|l|}{ Valores de laboratorio } \\
\hline Tasa de filtrado glomerular & $4,00(2,9$ a 6,2$)$ & $4,37(3,4$ a 6,5$)$ & $4,00(2,8$ a 6,0$)$ & 0,13 \\
\hline Hemoglobina (g/dL) & $7,2 \pm 2,6$ & $7,4 \pm 2,7$ & $7,10 \pm 2,53$ & 0,44 \\
\hline $\mathrm{pH}$ & $7,24(7,2$ a 7,4$)$ & $7,3(7,2$ a 7,3$)$ & $7,30(7,2$ a 7,4$)$ & 0,02 \\
\hline $\mathrm{HCO} 3(\mathrm{mEq} / \mathrm{L})^{*}$ & $10,9 \pm 4,7$ & $10,2 \pm 4,7$ & $11,2 \pm 4,7$ & 0,20 \\
\hline Potasio $(\mathrm{mEq} / \mathrm{L})^{*}$ & $6,2 \pm 1,5$ & $5,9 \pm 1,6$ & $6,3 \pm 1,4$ & 0,17 \\
\hline Calcio (mg/dl) & $7,70(7,0$ a 8,5$)$ & $7,90(7,0-8,4)$ & $7,70(6,7-8,4)$ & 0,48 \\
\hline Fósforo $(\mathrm{mg} / \mathrm{dL})^{*}$ & $7,3 \pm 2,9$ & $7,4 \pm 2,6$ & $7,2 \pm 2,9$ & 0,67 \\
\hline
\end{tabular}

*Media \pm desviación estándar mediana (rango intercuartílico)

ERC: enfermedad renal crónica HN2M: Hospital Dos de Mayo

promedio de edad de 56,86 \pm 15,48 años y la mayoría de ellos fueron de sexo masculino. La nefropatía diabética fue la etiología más frecuente de ERC en el $39 \%$ de los casos. En 53 participantes no se encontró información acerca de la etiología de ERC.

Cerca de la mitad de los participantes habían sido diagnosticados con ERC hace menos de un mes. Casi todos los pacientes tuvieron como primer acceso vascular un catéter venoso central no tunelizado. Solo un tercio de los participantes del estudio habían sido atendidos previamente en el HN2M. Casi el $80 \%$ de los participantes no habían sido evaluados previamente por un nefrólogo, según nuestra definición (Tabla 1). La mayoría de pacientes tenían anemia severa, acidosis y alteraciones como hipercalcemia, hipocalcemia e hiperfosfatemia.

La mediana del seguimiento en los participantes fue 1,7 (RIC 1 a 3) semanas. Hubo 51 (24\%) muertes durante la estancia intrahospitalaria. La tasa de mortalidad fue de 9,3 muertes por 100 personas-semanas (IC 95\%:
$7,0$ a 12,3$)$. La supervivencia a las cuatro semanas de seguimiento fue $74,5 \%$ (IC 95\%: 64,9 a 81,8 ), a las ocho semanas fue $46,01 \%$ (IC 95\%: 29,51 a 61,03) y las doce semanas fue 18,4\% (IC 95\%: 3,6 a 41,9) (Figura 1).

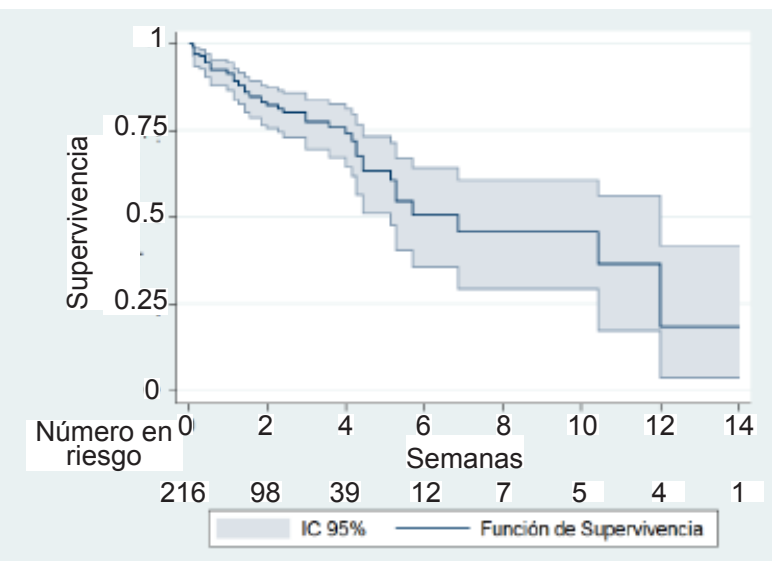

Figura 1. Curva de Kaplan-Meier de los participantes del estudio 


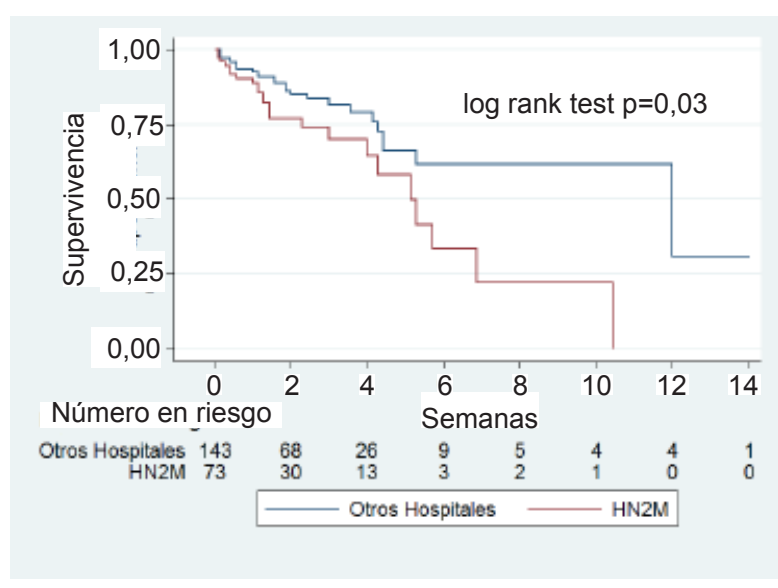

Figura 2. Curvas de Kaplan-Meier de los pacientes procedentes de otros hospitales versus los del Hospital Nacional Dos de Mayo (HN2M).

Respecto a los parámetros clínicos según el estado vital de los participantes no hubo mayores diferencias significativas, excepto para las variable $\mathrm{pH}$ y el haber sido atendido previamente en el HN2M (Tabla 1). Se evidenció una mediana menor de $\mathrm{pH}$ en los participantes fallecidos comparado con los vivos $(7,26$ versus 7,30 ; $p=0,02)$. El no haber sido atendido previamente en el HN2M estuvo relacionado con mayor mortalidad (log rank test $p=0,03$ ) (Figura 2). Sin embargo, no hubo diferencias en la función de supervivencia entre los pacientes con atención previa por nefrólogo y los que no tuvieron dicha atención (Figura 3).

En el modelo de regresión logística multivariado no se encontró asociación significativa entre las variables planteadas a priori y la mortalidad intrahospitalaria (Tabla 2). No se evidenció un significativo mayor riesgo de morir entre los pacientes diabéticos comparados con los de otras etiologías. De igual forma, a pesar de no ser significativo,

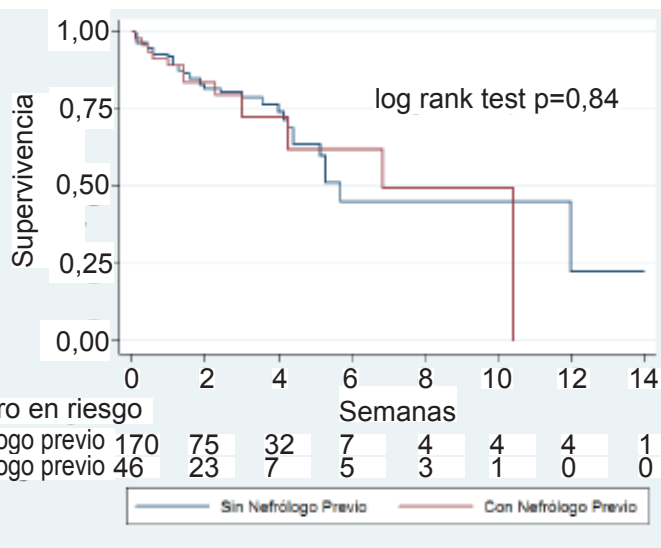

Figura 3. Curvas de Kaplan-Meier de pacientes sin atención previa por nefrólogo versus aquellos pacientes con atención previa por nefrólogo
Tabla 2. Modelo de regresión logística multivariado entre variables clínico-demográficas y estado vital

\begin{tabular}{|c|c|c|}
\hline Variable & $\begin{array}{l}\text { Odds Ratio ajustado } \\
\text { (IC } 95 \%)\end{array}$ & Valor $p$ \\
\hline Edad & $1,02(0,98$ a 1,05$)$ & 0,33 \\
\hline \multicolumn{3}{|l|}{ Etiología } \\
\hline Nefropatía diabética & 1,00 & -- \\
\hline Glomerulonefritis crónica & $0,88(0,26$ a 2,97$)$ & 0,64 \\
\hline Uropatía obstructiva & $0,28(0,06$ a 0,99$)$ & 0,04 \\
\hline Nefropatía hipertensiva & $0,36(0,09$ a 1,53$)$ & 0,17 \\
\hline Poliquistosis renal & $0,33(0,04$ a 3,17$)$ & 0,34 \\
\hline \multicolumn{3}{|l|}{ Tiempo de enfermedad } \\
\hline$<1$ mes & 1,00 & -- \\
\hline 1 a 6 meses & $0,84(0,32$ a 2,26$)$ & 0,74 \\
\hline$>6$ meses & $0,55(0,19$ a 1,57$)$ & 0,26 \\
\hline Atendido por nefrólogo & $1,14(0,39$ a 3,31$)$ & 0,85 \\
\hline $\begin{array}{l}\text { Logaritmo de tasa de } \\
\text { filtrado glomerular }\end{array}$ & $0,80(0,38$ a 1,69$)$ & 0,56 \\
\hline
\end{tabular}

se encontró una tendencia a menor riesgo de fallecer en los pacientes que tenían entre un mes y seis meses con el diagnóstico de ERC, y en aquellos con más de seis meses con el diagnostico de ERC comparado con aquellos que tenían menos de un mes con ese diagnóstico [OR=0,84 IC $95 \%(I C ~ 95 \% 0,32$ a 2,26) y OR=0,55 (IC 95\% 0,19 a 1,57), respectivamente]. Así mismo, los pacientes atendidos previamente por un nefrólogo presentaron mayor riesgo de fallecer OR= 1,14 (IC 95\% 0,39 a 3,31), aunque esta diferencia no fue significativa.

\section{DISCUSIÓN}

Los principales hallazgos de este estudio muestran que uno de cada cuatro pacientes incidentes en HD falleció durante su primera hospitalización. Se encontró una tendencia a que los pacientes que tuvieron mayor tiempo con diagnóstico de ERC tuvieran menor mortalidad. Así mismo, evidenciamos que los pacientes atendidos previamente por un nefrólogo no tenían diferencias en la mortalidad al ser comparados con aquellos que no recibieron dicho cuidado previo.

Los costos de la atención de pacientes en diálisis varían de acuerdo al país, pero son una carga muy alta para los sistemas de salud. Por ejemplo, en el 2010 EE. UU. gastó 77506 dólares al año por paciente; Brasil, el mismo año, gastaba entre 7980 y 13428 dólares y Sri Lanka entre 5869 y 8804 dólares por paciente al año (11). Desafortunadamente, no todos los países cuentan con recursos para dar cobertura total a todos los pacientes que requieren diálisis. En México, solo el $22 \%$ de los pacientes con ERC recibió alguna forma de TRR (HD, diálisis peritoneal o trasplante) en el año 2009, lo que representó un costo aproximado de 559 millones dólares anuales en insumos para los procedimientos. Si se hubiera atendido a 
todas las personas con ERC, se hubiera requerido más de 2400 millones de dólares anuales ${ }^{(13)}$.

Esta falta de recursos económicos en los sistemas de salud para dar una cobertura total a los pacientes en HD ha dado como consecuencia mortalidades alarmantes en países africanos ${ }^{(14,15)}$, lo que en Nigeria ha llevado a preguntarse si vale la pena iniciar HD en pacientes que no podrán costearse la terapia completa ${ }^{(14)}$. En nuestro país, el MINSA no cuenta con recursos para dar cobertura total a todos los pacientes que requieren $\operatorname{HD}{ }^{(7,8)}$, así como no tiene un programa integral para la evaluación en estados prediálisis, como sí ocurre en otras partes del mundo ${ }^{16}$. Esto es relevante ya que se ha sugerido que una inadecuada preparación previa a la diálisis está relacionada con mayor mortalidad ${ }^{(17,19)}$. De tal forma que los factores asociados a la mortalidad temprana nos puede dar una idea de las condiciones en las que los pacientes llegan a diálisis y ser una forma indirecta de evaluar nuestro sistema de salud para con nuestros pacientes con ERC ${ }^{(4,5)}$.

El hallazgo de que casi la mitad de nuestros pacientes ingresan a diálisis con el diagnóstico de la ERC hecho hace menos de un mes, nos demuestra que el diagnóstico precoz de la enfermedad es una de las tareas pendientes en el sistema de salud peruano. El despistaje de la ERC mediante la albuminuria y el cálculo de la TFG son medidas económicas y costoefectivas en la prevención primaria de la enfermedad en las poblaciones de riesgo como los pacientes con diabetes mellitus ${ }^{(20,21)}$, quienes en nuestra población tuvieron una tendencia a mayor mortalidad.

El diagnóstico precoz de la enfermedad nos permitirá instaurar medidas de disminución de la progresión de la ERC o de preparación para el ingreso en buenas condiciones a las TRR ${ }^{(20)}$. Hemos encontrado una tendencia entre mayor tiempo de diagnóstico de la ERC y evaluación previa en nuestro hospital con menor mortalidad, esta última característica solo presente en uno de cada tres de nuestros pacientes. La mayoría, o son pacientes que no habían tenido evaluaciones médicas previas, o pertenecían a otros hospitales y llegaron a nuestro centro al carecer estos o de centros de HD o de cupos disponibles para HD en ellos ${ }^{(7)}$. Esto refuerza la idea de la necesidad de una reestructuración del sistema de atención con la finalidad de dar cobertura total a los pacientes de con ERC en el MINSA.

Hemos encontrado que no hay diferencias en la mortalidad entre los pacientes con evaluaciones previas por un nefrólogo, las que, contrariamente, se han relacionado con mejores resultados en diálisis en otros reportes ${ }^{(22-26)}$. Es probable que los pacientes evaluados por un nefrólogo en nuestro hospital estén en peor condición clínica, de tal forma que eso explique que no haya diferencias con aquellos pacientes sin control previo. De igual forma, quizá una evaluación nefrológica previa no sea, por sí misma, un factor protector si es que esta no encausa medidas terapéuticas correctas, las que no siempre se consiguen tanto por incumplimiento de pacientes, como por trabas burocráticas, inadecuada atención del nefrólogo ${ }^{(27)}$, e incluso, del número de evaluaciones del mismo ${ }^{(6)}$.

Del mismo modo, la mortalidad en las primeras semanas de HD, depende también de la situación clínica de las otras enfermedades de los pacientes al llegar a diálisis $o$ al ser referidos al nefrólogo. No es infrecuente que estas estén poco controladas, como hemos reportado previamente en pacientes diabéticos que acuden por primera vez a consultorios de nefrología en hospitales del MINSA de Lima ${ }^{(28)}$.

Este trabajo tiene importantes limitaciones. Primero, no se tiene definidas las causas de mortalidad, y el análisis se ha conducido usando "todas las causas de mortalidad". Segundo, el número pequeño de participantes y el tener una alta frecuencia de factores asociados a mal control previo, tanto en la población fallecida como en la viva, harían más difícil encontrar las diferencias entre las asociaciones de estos factores. Tercero, al no tener datos específicos respecto al historial médico de origen entre los pacientes sin historia clínica en el HN2M, no fue posible estratificarlos de acuerdo a su hospital y fueron juntados en un solo grupo, dentro de los que pueden haber tanto atendidos en otros hospitales o incluso sin atención médica previa. Finalmente, nuestro estudio es unicéntrico, y sus hallazgos no pueden extrapolarse a otros hospitales del MINSA; sin embargo, por estar hecho en un hospital de referencia nacional podría servir para conocer aspectos de la mortalidad temprana en los pacientes con ERC.

En conclusión, se ha encontrado una deficiente atención previa en los pacientes con ERC y que están asociadas a un aumento de mortalidad intrahospitalaria.

Contribuciones de autoría: PHA, AVH, VABZ han participado en la concepción del artículo, PHA hizo la recolección de datos AHV realizó el análisis de datos, PHA y AVH hicieron la redacción y todos se encargaron de la revisión crítica y aprobación

Agradecimientos: al Dr. Javier Hernández Pacheco jefe del Servicio de Nefrología del Hospital Nacional 2 de Mayo.

Fuentes de financiamiento: autofinanciado.

Conflictos de interés: los autores declaran no tener conflictos de interés en la publicación de este artículo. 


\section{REFERENCIAS BIBLIOGRÁFICAS}

1. Nordio M, Limido A, Maggiore U, Nichelatti M, Postorino M, Quintaliani $\mathrm{G}$, et al. Survival in patients treated by long-term dialysis compared with the general population. Am J Kidney Dis. 2012 Jun;59(6):819-28. doi: 10.1053/j. ajkd.2011.12.023.

2. Goodkin DA, Bragg-GreshamJL, Koenig KG, Wolfe RA, Akiba T, Andreucci VE, et al. Association of comorbid conditions and mortality in hemodialysis patients in Europe, Japan, and the United States: the Dialysis Outcomes and Practice Patterns Study (DOPPS). J Am Soc Nephrol. 2003;14(12):3270-7.

3.- Robinson BM, Zhang J, Morgenstern $\mathrm{H}$, Bradbury BD, Ng LJ, et al. Worldwide, mortality risk is high soon after initiation of hemodialysis. Kidney Int. 2014;85(1):158-65. doi: 10.1038/ ki.2013.252

4.- Foley RN, Chen SC, Solid CA, Gilbertson DT, Collins AJ. Early mortality in patients starting dialysis appears to go unregistered. Kidney Int. 2014;86(2):392-8. doi: 10.1038/ ki.2014.15.

5.- Rognant N, Laville M. Early mortality in dialysis and adequacy of predialysis renal care: the picture is more complex than we thought. Kidney Int. 2014;86(2):238-40. doi: 10.1038/ki.2014.82.

6.- Singhal R, Hux JE, Alibhai SM, Oliver MJ. Inadequate predialysis care and mortality after initiation of renal replacement therapy. Kidney Int. 2014;86(2):399406. doi: 10.1038/ki.2014.16.

7.- Herrera-Añazco P, Mezones-Holguín E, Hernandez AV. Global Kidney Disease. Lancet. 2013;382(9900):1243-4. doi: 10.1016/S0140-6736(13)62087-5.

8.- Herrera-Añazco P, Palacios-Guillén $M$, Hernandez AV. Alta tasa de interrupcion de hemodiálisis en pacientes del hospital nacional 2 de mayo de Perú. Rev Nefrol Dial Tras. 2014;34(2):94-8.

9.- Herrera-Añazco P, Palacios-Guillén $M$, Chipayo-Gonzales D, Silveira-Chau M. Mortalidad durante la primera hospitalización en una población que inicia diálisis crónica en un hospital general. An Fac Med. 2013;74(3):199-202.

10.- National Kidney Fundation. K/DOQI clinical practice guidelines for chronic kidney disease: evaluation, classification, and stratification. Am J Kidney Dis. 2002;39(2 Suppl 1):S1-266.
11.- Ranasinghe P, Perera YS, Makarim MF, Wijesinghe A, Wanigasuriya $\mathrm{K}$. The costs in provision of haemodilysis in a developing country: a multy - centered study. BMC Nephrol. 2011;12:42. doi: $10.1186 / 1471-2369-12-42$.

13.- Subsecretaria de Innovación y Calidad. Red Estratégica de Servicios de Salud contra La Enfermedad Renal Crónica en México. Mexico D.F.: Vivir Mejor; 2010.

14.- Ekrikpo UE, Udo AI, Ikpeme EE, Effa EE. Haemodialysis in an emerging centre in a developing country: a two year review and predictors of mortality. BMC Nephrol. 2011;12:50. doi: 10.1186/1471-2369-12-50.

15.- Shibiru T, Gudina EK,Habte B, Derbew A, Agonafer T. Survival patterns on maintenance hemodialysis for end stage renal disease in Ethiopia: summary of 91 cases. BMC Nephrol. 2013;14:127. doi: 10.1186/1471-2369-14-127.

16.- Otero González A. Guías SEN para el manejo de la enfermedad renal crónica avanzada y prediálisis. Nefrologia. 2008; $28 \operatorname{Supl}(3): 1$.

17.- Roderick P, Jones C, Drey N, Blakeley S, Webster P, Goddard J, et al. Late referral for end-stage renal disease: a region wide survey in the south west of England. Nephrol Dial Trasplant. 2002;17(7):1252-9.

18.- Cass A, Cunningham J, Arnold PC, Snelling P, Wang Z, Hoy W. Delayed referral to a nephrologist: outcomes among patients who survive at least on year on dialysis. Med J Aust. 2002;177(3):135-8.

19.- Stack AG. Impact of timing of nephrology referral and pre-ESRD care on mortality risk among new ESRD patients in the United States. Am J Kidney Dis. 2003;41(2):310-8.

20.- Stevens PE, Levin A; Kidney Disease: Improving Global Outcomes Chronic Kidney Disease Guideline Development Work Group Members. Evaluation and management of chronic kidney disease: synopsis of the kidney disease: improving global outcomes 2012 clinical practice guideline. Ann Intern Med. 2013;158(11):825-30 doi: $\quad 10.7326 / 0003-4819-158-11$ 201306040-00007.

21.- KDOQI. KDOQI clinical practice guidelines and clinical practice recommendations for diabetes and chronic kidney disease. Am J Kidney Dis. 2007;49(2 Suppl 2):S12-154

22.- Frimat L, Loos-Ayav C, Panescu V, Cordebar N, Briançon S, Kessler M. Early referral to a nephrologist is associated with better outcomes in type 2 diabetes patients with end-stage renal disease. Diabetes Metab. 2004;30(1):67-74

23. Maynard C, Cordonnier D. [The late referral of diabetic patients with kidney insufficiency to nephrologists has a high human and financial cost: interdisciplinary communication is urgently needed]. Diabetes Metab. 2001;27(4 Pt 1):517-21. [Articulo en frances]

24. Saggi SJ, Allon M, Bernardini J, KalantarZadeh K, Shaffer R, Mehrotra R, et al. Considerations in the optimal preparation of patients for dialysis. Nat Rev Nephrol. 2012;8(7):381-9. doi: 10.1038/nrneph.2012.66.

25. Smart NA, Titus TT. Outcomes of early versus late nephrology referral in chronickidneydisease: asystematicreview. Am J Med. 2011;124(11):1073-80.e2. doi: 10.1016/j.amjmed.2011.04.026.

26. Smart NA, Dieberg G, Ladhani M, Titus T. Early referral to specialist nephrology services for preventing the progression to end-stage kidney disease. Cochrane Database Syst Rev. 2014;6:CD007333. doi: $\quad 10.1002 / 14651858 . C D 007333$. pub2.

27.- Hughes SA, Mendelssohn JG, Tobe SW, McFarlane PA, Mendelssohn DC. Factors associated with suboptimal initiation of dialysis despite early nephrologist referral. Nephrol Dial Transplant. 2013;28(2):392-7. doi: 10.1093/ndt/ gfs 431 .

28.- Herrera-Añazco P, Bonilla-Vargas L, Palacios-Guillén M, Valencia-Rodríguez J, Sánchez-Riva F, Salomé-Luna J, et al. Características clínicas de los pacientes diabéticos que acuden por primera vez a una consulta nefrológica en hospitales públicos de Lima. An Fac Med. 2014;75(1):25-9.

Correspondencia: Percy Herrera Añazco Dirección: Olavegoya 1879 dpto. 701 Jesús

Maria - Lima

Teléfono: (511) 993457515

Correo electrónico: silamud@gmail.com 